\title{
Managing work-related mental health conditions in family medicine
}

\author{
Michael Kidd AM MD
}

Cite as: CMAJ 2019 March 25;191:E323-4. doi: 10.1503/cmaj.190284

$\mathrm{n}$ n 2016, the Mental Health Commission of Canada estimated that in any given year, 1 in 5 people in Canada has a mental health problem or illness. ${ }^{1,2}$ Many will face mental health conditions during their working years, and work-related mental health conditions are estimated to be responsible for about 30\% of disability claims, costing the Canadian economy more than $\$ 6$ billion each year in lost productivity, from absenteeism, presenteeism and staff turnover. ${ }^{1,2}$ Patients whose mental illness is work-related may submit a worker's compensation claim.

In Canada, family physicians are the health care providers most commonly seen for mental health conditions. ${ }^{3}$ Family physicians may be asked to provide information and advice to compensation boards on issues such as the ability of a person to return to work and how much a patient's mental health condition is work related. ${ }^{3} \mathrm{~A}$ recently published Australian evidencebased clinical practice guideline aims to support family physicians to diagnose and manage patients with work-related mental health conditions. ${ }^{4}$

Participation in safe work conditions is a key component for many people in achieving good overall health. When a mental health condition arises because of factors related to the work environment, negative consequences can be compounded unless they are addressed appropriately and promptly. ${ }^{4}$ Yet, unlike many acute physical injuries, which are often visible, work-related mental health conditions can be difficult to diagnose and treat, and patients with these conditions often have the longest duration off work as they recover. Some of the challenges facing family physicians include delineating how a condition is related to work, for example, proving if it is an exacerbation of a pre-existing mental health condition secondary to work or as a result of work, or if the mental illness has developed secondary to a primary physical condition that is the focus of a compensation claim. ${ }^{1,5}$ Until now there has been little guidance for physicians in addressing these issues.

A group of Australian researchers and clinicians, led by a family physician, responded to a call-to-action by family physicians to develop the new guideline. ${ }^{4}$ Although the Australian and Canadian health care systems differ in important ways, diagnostic uncertainties and system complexities faced by family physicians are similar. And like Canadian family physicians, Australian family physicians are relied on by patients to provide information about the

\section{KEY POINTS}

- Many people face mental health problems or illness during their working years; some mental health conditions may be work related and lead to submission of a worker's compensation claim.

- Diagnosing and managing a work-related mental health condition is often not straightforward.

- A new Australian evidence-based guideline aims to support family physicians who treat patients with work-related mental health conditions such as depression, anxiety, posttraumatic stress disorder and substance use disorder.

compensation process, and by compensation schemes to make judgements about the work-relatedness of a mental health condition and a patient's capacity to continue working or return to work. ${ }^{6}$

Drawn from Australian and international sources, and developed according to the standards and procedures of the Australian Government's National Health and Medical Research Council, the new clinical guideline covers the full range of work-related mental health conditions, including depression, anxiety, posttraumatic stress disorder, acute stress disorder, adjustment disorder and substance misuse disorder. ${ }^{1}$ It is the first of its kind anywhere in the world, despite work-related mental health conditions being the second most common cause of workers' compensation claims, at least in Australia, after musculoskeletal disorders. ${ }^{6}$

The guideline, which is structured using a clinical reasoning pathway, includes 11 evidence-based recommendations and, where clear evidence is not available, 19 consensus-based recommendations. The guideline provides tools to assist assessment and diagnosis, and includes recommendations on the work-relatedness of mental health conditions, patient management including decisions about return to work, comorbid or secondary mental health conditions, conveying a diagnosis to a patient and communication with others in the workplace. ${ }^{4}$ It promotes collaboration, communication, cultural sensitivity and appropriate care, highlighting the important role that can be played by family physicians, and the members of their teams, in each person's personal recovery and return to work. 
With increased awareness and the growing burden of mental health conditions in our community, family physicians need to be equipped with evidence-based recommendations, tools and resources to support decision-making at the point of care. After all, over the course of a lifetime, $43 \%$ of Canadians will have a mental health problem or illness, ${ }^{1,2}$ and many of these will arise while a person is working. In the current absence of a Canadianspecific guideline, this new resource may provide some practical recommendations to support optimal patient care.

The new guideline is available at www.monash.edu/ work-related-mental-health-guideline.

\section{References}

1. Making the case for investing in mental health in Canada. Ottawa: Mental Health Commission of Canada; 2016.

2. Smetanin $\mathrm{P}$, Stiff D, Briante $\mathrm{C}$, et al. The life and economic impact of major mental illnesses in Canada: 2011 to 2041. Toronto: RiskAnalytica on behalf of the Mental Health Commission of Canada; 2011.

3. Lesage A, Vasiliadis H-M, Gagné M-A, et al. Prevalence of mental illnesses and related service utilization in Canada: an analysis of the Canadian Community Health Survey. Mississauga (ON): Canadian Collaborative Mental Health Initiative; 2006. Available: www.shared-care.ca/09_Prevalence_EN.pdf (accessed 2019 Mar. 13).
4. Mazza D, Brijnath B, Chakraborty SP; Guideline Development Group. Clinical guideline for the diagnosis and management of work-related mental health conditions in general practice. Melbourne (Australia): Monash University; 2018. Available: www.monash.edu/work-related-mental-health-guideline (accessed 2019 Mar. 13).

5. Kosny A, Lifshen M, Tonima S, et al. The role of health-care providers in the workers' compensation system and return-to-work process: final report. Toronto: Institute for Work \& Health; 2016.

6. Brijnath B, Mazza D, Singh N, et al. Mental health claims management and return to work: qualitative insights from Melbourne, Australia. J Occup Rehabil 2014;24:766-76.

\section{Competing interests: None declared.}

This article was solicited and has not been peer reviewed.

Affiliations: Department of Family and Community Medicine, Faculty of Medicine, University of Toronto; Institute for Health System Solutions and Virtual Care, Women's College Hospital, Toronto, Ont.; Murdoch Children's Research Institute, Royal Children's Hospital, Melbourne, Australia; Southgate Institute for Health, Society and Equity, Flinders University, Adelaide, Australia

Correspondence to: Michael Kidd, michael.kidd@utoronto.ca 